\title{
Sintomas de ansiedade e depressão entre estudantes de medicina: estudo de prevalência e fatores associados
}

Symptoms of anxiety and depression among medical students: study of prevalence and associated factors

\author{
Bartira Oliveira Sacramento' (1) bartiraos@gmail.com \\ Tassiana Lima dos Anjos' 1 (1) tassianaanjos15.2@bahiana.edu.br \\ Ana Gabriela Lopes Barbosa' (1) anabarbosa15.2@bahiana.edu.br \\ Camila Fagundes Tavares' (1) camilatavares15.2@bahiana.edu.br \\ Juarez Pereira Dias' 1 | juarezdias@bahiana.edu.br
}

\section{RESUMO}

Introdução: Os transtornos mentais comuns (TMC) implicam sofrimento psíquico e interferem nas atividades diárias, nos relacionamentos interpessoais e na qualidade de vida. Estima-se que os TMC atinjam de $9 \%$ a $12 \%$ da população mundial e de $12 \%$ a $15 \%$ da brasileira em todas as faixas etárias. Dentre os diferentes grupos sociais, os estudantes universitários possuem maior vulnerabilidade para desenvolver transtornos de ansiedade e depressão.

Objetivo: Diante disso, este estudo se propôs a estimar a prevalência e os fatores associados a sintomas de ansiedade e depressão em estudantes de Medicina de uma capital do Nordeste brasileiro.

Métodos:Trata-se de um estudo de prevalência, com uma amostra probabilística dos 1.339 alunos que frequentavam regularmente os 12 semestres do curso de Medicina em janeiro de 2018. Os dados foram coletados por meio da aplicação de questionário socioeconômico, comportamental e demográfico e dos Inventários de Ansiedade e de Depressão de Beck. Utilizou-se o teste de qui-quadrado para verificação de diferenças entre sintomas de ansiedade e depressão e variáveis socioeconômicas e comportamentais e as prevalências (total e por nível de gravidade) e a razão de prevalência (RP) bruta e ajustada como medida de associação. A análise de tendência linear foi empregada para verificar a existência de relação entre sintomas de ansiedade e depressão e semestres do curso. As variáveis que apresentaram RP bruta com $p<0,20$ foram incorporadas na análise multivaridada, no modelo de regressão de Poisson robusto, para determinação da RP ajustada.

Resultados: Quanto à prevalência de sintomas, constatou-se o seguinte: $30,8 \%$ para ansiedade e $36,0 \%$ para depressão. A RP bruta e ajustada para sintomas de ansiedade teve associação estatisticamente significante para sexo, idade e orientação sexual. A RP bruta e ajustada para sintomas de depressão teve associação estatisticamente significante para sexo, raça/cor da pele e orientação sexual. As análises de correlação entre os semestres do curso e a presença de sintomas de ansiedade e depressão indicaram fraco coeficiente de determinação, caráter descendente e sem significância estatística.

Conclusões: Por se tratar de um estudo de prevalência, esta investigação não possibilita conclusões sobre causalidade. Estudos de acompanhamento adicionais são necessários para elucidar o curso da ansiedade e depressão ao longo dos semestres letivos.

Palavras-chave: Ansiedade; Depressão; Estudantes de Medicina; Ensino Médico.

\section{ABSTRACT}

Introduction: Common Mental Disorders (CMDs) imply psychological distress, interfering with daily activities, interpersonal relationships and quality of life. It is estimated that CMDs affect 9\% to $12 \%$ of the world's population and $12 \%$ to $15 \%$ of the Brazilian population in all age groups. Among different social groups, university students are more vulnerable to the development of anxiety and depression disorders.

Objective: Therefore, this study proposed to estimate the prevalence rates and factors associated with symptoms of anxiety and depression in medical students in a capital city of northeast Brazil.

Methods: This is a prevalence study, with a probabilistic sample of 1,339 students who regularly attended the 12 semesters of medical school in January 2018. Data were collected by applying a socioeconomic, behavioral and demographic survey and Beck Anxiety and Depression Inventories. The chi-square test was used to check for differences between anxiety and depression symptoms and socioeconomic and behavioral variables. The prevalence rates (total and by level of severity) and the crude and adjusted prevalence ratio (PR) were used as an association measure. Linear trend analysis was used to verify the existence of an association between anxiety and depression symptoms and semesters of the medical school. The variables that showed a crude PR with $p<0.20$ were incorporated into the multivariate analysis, using the robust Poisson regression model, to determine the adjusted PR.

Results: The prevalence of symptoms of anxiety was 30.8\%, whereas depression was $36.0 \%$. The crude and adjusted PR for anxiety symptoms showed a statistically significant association with gender, age and sexual orientation. The crude and adjusted PR for symptoms of depression showed a statistically significant association with gender, ethnicity/skin color and sexual orientation. The correlation analyses between the semesters of the course and the presence of anxiety and depression symptoms indicated a weak coefficient of determination, with a descending characteristic and without statistical significance.

Conclusions: As this is a prevalence study, this investigation does not allow conclusions on causality. Additional follow-up studies are needed to elucidate the course of anxiety and depression throughout the school semesters.

Keywords: Anxiety; Depression; Medical Students; Medical Education.

'Escola Bahiana de Medicina e Saúde Pública, Salvador, Bahia, Brasil.

Editora-chefe: Daniela Chiesa

| Editor associado: Roberto Zonato Esteves

Recebido em 04/09/20; Aceito em 06/12/20. | Avaliado pelo processo de double blind review. 


\section{INTRODUÇÃO}

Os transtornos mentais comuns (TMC) correspondem a quadros clínicos em que o indivíduo apresenta sintomatologia de ansiedade, depressão, tristeza, fadiga, insônia, estresse, irritabilidade, e queixas somáticas, como anorexia, falta de ar, cefaleia, ente outras ${ }^{1-3}$. Todavia, essas manifestações não preenchem critérios suficientes para diagnóstico psíquico formal de depressão e/ou ansiedade segundo as classificações da quinta edição do Manual diagnóstico e estatístico de transtornos mentais: DSM-5 e da Classificação Internacional de Doenças (CID-11) 4,5 , embora apresentem intensidade e frequência capazes de implicar sofrimento psíquico para os indivíduos, de modo a interferir nas atividades diárias deles, nos relacionamentos interpessoais e na qualidade de vida ${ }^{1,2,6,7}$.

Estima-se que os TMC atinjam de $9 \%$ a $12 \%$ da população mundial e de $12 \%$ a $15 \%$ da brasileira em todas as faixas etárias $^{8}$. Segundo a Organização Mundial da Saúde (OMS), esse quantitativo vem aumentando em todo mundo, principalmente em países de baixa renda, em indivíduos do sexo feminino, mais pobres e desempregados, justamente aqueles nos quais os sintomas de ansiedade e depressão são mais frequentes ${ }^{9-11}$. No Brasil, a prevalência de TMC, segundo diversos autores, varia de $19,7 \%$ a $43,7 \%$, o que representa uma parcela significativa da população que pode requer atendimento especializado ${ }^{12,13}$.

Dentre os diferentes grupos sociais, os estudantes universitários possuem maior vulnerabilidade para desenvolver transtornos de ansiedade e depressão, e já existem estudos que utilizam diferentes instrumentos, tais como o Self Reporting Questionnaire (SRQ-20) ${ }^{14}$, Beck Anxiety Inventory (BAI) ${ }^{15}$ e Beck Depression Inventory $(\mathrm{BDI})^{16}$, que demonstram alta prevalência desses transtornos, sobretudo em estudantes de Medicina de vários continentes ${ }^{16,17}$. Em países em desenvolvimento econômico, como o Egito, um estudo realizado com 700 estudantes de Medicina no ano de 2017 reportou uma alta prevalência de ansiedade (73\%) e depressão (65\%) ${ }^{18}$. NaTurquia, constataram-se os seguintes resultados: $35,8 \%$ para sintomas de ansiedade média e moderada e 30,5\% para depressão média e moderada e $8,5 \%$ para grave ${ }^{19}$.

Por sua vez, no Brasil em 2015, estudos realizados com universitários de Medicina de Santa Catarina revelaram prevalências de $35,5 \%$ e 32,8\%, respectivamente para ansiedade e depressão ${ }^{20}$. Já na Faculdade Pernambucana de Saúde (FPS), em 2014, a prevalência foi de 19,7\% para ansiedade e 5,6\% para depressão ${ }^{21}$.

Diante desse cenário de alta frequência de TMC em estudantes universitários, o objetivo deste estudo foi estimar a prevalência e os fatores associados a sintomas de ansiedade e depressão em estudantes de Medicina de uma capital do Nordeste brasileiro em 2018.

\section{MÉTODOS}

Trata-se de um estudo de prevalência, com uma amostra probabilística dos 1.339 alunos que frequentavam regularmente todos os 12 semestres do curso de Medicina em janeiro de 2018 e que assinaram o Termo de Consentimento Livre e Esclarecido/ Termo de Assentimento. Para o cálculo amostral, consideraramse como parâmetros a prevalência média esperada de sintomas de ansiedade e depressão em estudantes universitários de $35 \%^{20,22,23}$, o erro alfa aceitável de $5 \%(a=5 \%$ ), o efeito do desenho de 1,5 e o nível de confiança de $95 \%$. Foi estimado em 417 o número de alunos que, acrescidos de $10 \%$ de perdas e recusas, totalizou 457, divididos proporcionalmente, o que resultou em uma média de 35 discentes por semestre, os quais foram selecionados mediante sorteio aleatório simples.

Os dados foram coletados por meio da aplicação dos seguintes instrumentos: 1. questionário estruturado para registro de informações sociodemográficas, econômicas e comportamentais; 2. Inventário de Ansiedade de Beck (BAI) desenvolvido por Beck et al. ${ }^{15}$ e traduzido para o português e validado no Brasil em estudantes universitários por Quintão et al. ${ }^{24}$; e 3. Inventário de Depressão de Beck (BDI) também elaborado por Beck et al. ${ }^{16}$ e validado na língua portuguesa, no Brasil, por Andrade et al. ${ }^{25}$ e Gomes-Oliveira et al. ${ }^{26}$. Segundo os pontos de corte estabelecidos pelo BAl, considerou-se a seguinte pontuação: < 10 para ausência ou sintomas de ansiedade mínima, 11-19 para leve, 20-30 para moderada e 30-63 para severa. No caso do BDI, considerou-se a seguintes pontuação para depressão: < 10 para ausente ou mínima, 10-18 para leve, 19-29 para moderada e 30-63 para severa. Para esse estudo, considerou-se como presença de sintomas de ansiedade e depressão a pontuação > 10.

Realizaram-se análises descritivas mediante distribuição das frequências absolutas ( $\mathrm{n}$ ) e relativas (\%) dos estratos das variáveis de interesse. Para verificação de possíveis diferenças entre sintomas de ansiedade e depressão e variáveis sociais, econômicas e comportamentais, foi utilizado o teste de quiquadrado (x2) de Pearson. E como medida de associação, utilizaram-se as prevalências (total e por nível de gravidade) e a razão de prevalência (RP) bruta e ajustada (Aju). Para verificação de diferença entre as prevalências, adotou-se o teste KruskalWallis, assumindo como significância estatística $p<0,05$.

As variáveis que apresentaram RP bruta com $p<0,20$ foram incorporadas na análise multivaridada, no modelo de regressão de Poisson robusto, para determinação da RP Aju. Os dados foram processados e analisados nos softwares SPSS versão 22 e Stata versão 15.1. O projeto desta pesquisa foi aprovado pelo Comitê de Ética em Pesquisa da Escola Bahiana de Medicina e Saúde Pública (EBMSP), sob os nºs. 2.519 .431 de 28 de fevereiro de 2018 e $n^{\circ} 2.572 .959$ de $1^{\circ}$ de abril de 2018 . 


\section{RESULTADOS}

Dos 458 universitários de Medicina (34,2\% do total) incluídos no estudo, $62,7 \%$ eram do sexo feminino e $60,0 \%$ tinham menos de 22 anos de idade. A mediana de idade para ambos os sexos foi de 22,0 anos. Os participantes se autodeclararam heterossexuais $(92,6 \%)$ e brancos $(56,7 \%)$, mencionaram ter namorado(a) fixo(a) (60,3\%) e afirmaram ser católicos (48,1\%). Em relação à renda familiar mensal, 50,1\% referiram menos de $\mathrm{R} \$ 8.000,00$. Quanto à procedência, 94,3\% eram do estado, sendo a grande maioria da capital $(61,3 \%)$. Moravam com familiares $81,1 \%$ (Tabela 1). Do total de alunos da amostra $11,1 \%$ cursavam o primeiro e décimo semestres do curso. A prevalência de sintomas de ansiedade foi de 30,8\%: leve $(91=19,9 \%)$, moderada $(39=8,5 \%)$ e severa $(11=2,4 \%)$. Sintomas mínimos foram identificados em 289 discentes $(63,1 \%)$ e ausentes em 28 (6,1\%). Quanto à depressão, a prevalência foi de $36,0 \%$ : leve $(132=28,8 \%)$, moderada $(27=5,9 \%)$ e severa ( 6 =1,3\%). Em 259 (56,6\%), os sintomas eram mínimos, e, em 34 (7,4\%), estavam ausentes (Gráfico 1).

A RP bruta da associação dos sintomas de ansiedade e sexo feminino, idade $\leq 22$ anos e homo/bissexuais e a RP bruta da associação dos sintomas de depressão e sexo feminino, homo/ bissexuais, raça/cor da pele outra (preta/parda), renda familiar $(<R \$$ $8.000,00$ ) e residir com outros (colegas/amigo, hotel/pensionato/

Tabela 1. Prevalência de sintomas de ansiedade e depressão em estudantes de Medicina segundo variáveis demográficas, sociais, econômica e comportamentais - Salvador-BA, 2018

\begin{tabular}{|c|c|c|c|c|c|c|c|c|c|c|}
\hline \multirow[t]{3}{*}{ Variável } & \multicolumn{5}{|c|}{ Ansiedade } & \multicolumn{5}{|c|}{ Depressão } \\
\hline & \multicolumn{2}{|c|}{ Sim } & \multicolumn{2}{|c|}{ Não } & & \multicolumn{2}{|c|}{ Sim } & \multicolumn{2}{|c|}{ Não } & \\
\hline & $\mathbf{n}$ & $\%$ & $\mathrm{n}$ & $\%$ & & $\mathbf{n}$ & $\%$ & $\mathrm{n}$ & $\%$ & \\
\hline \multicolumn{11}{|l|}{ Sexo } \\
\hline Feminino & 107 & 37,3 & 180 & 62,7 & $0,000^{*}$ & 123 & 42,9 & 164 & 57,1 & $0,000^{*}$ \\
\hline Masculino & 34 & 19,9 & 137 & 80,1 & & 42 & 24,6 & 129 & 75,4 & \\
\hline \multicolumn{11}{|l|}{ Idade (anos) } \\
\hline$\leq 22$ & 97 & 35,4 & 178 & 64,6 & $0,011^{*}$ & 100 & 36,5 & 174 & 63,5 & 0,798 \\
\hline$>22$ & 44 & 24,0 & 139 & 76,0 & & 65 & 35,3 & 118 & 64,7 & \\
\hline \multicolumn{11}{|l|}{ Orientação sexual } \\
\hline Homo/bissexual & 20 & 60,6 & 13 & 39,4 & $0,000^{*}$ & 21 & 63,6 & 12 & 36,4 & $0,001^{*}$ \\
\hline heterossexual & 121 & 28,5 & 303 & 71,5 & & 144 & 34,0 & 280 & 66,0 & \\
\hline \multicolumn{11}{|l|}{ Raça/cor da pele } \\
\hline Outras & 58 & 29,4 & 139 & 70,6 & 0,592 & 82 & 41,6 & 115 & 58,4 & $0,030^{*}$ \\
\hline Branca & 82 & 31,8 & 176 & 68,2 & & 82 & 31,8 & 176 & 68,2 & \\
\hline \multicolumn{11}{|l|}{ Situação afetiva } \\
\hline Com companhia fixa & 78 & 28,4 & 197 & 71,6 & 0,182 & 96 & 34,9 & 179 & 65,1 & 0,533 \\
\hline Sem companhia fixa & 62 & 34,3 & 119 & 65,7 & & 68 & 37,8 & 112 & 62,2 & \\
\hline \multicolumn{11}{|l|}{ Religião } \\
\hline Católicos & 62 & 28,3 & 157 & 71,7 & 0,274 & 70 & 32,0 & 149 & 68,0 & 0,081 \\
\hline Outros & 78 & 33,1 & 158 & 66,9 & & 94 & 39,8 & 142 & 60,2 & \\
\hline \multicolumn{11}{|l|}{ Renda familiar (R\$) } \\
\hline$\leq 12.000,00$ & 60 & 34,1 & 116 & 65,9 & 0,111 & 74 & 42,0 & 102 & 58,0 & $0,016^{*}$ \\
\hline$>12.000,00$ & 46 & 26,3 & 129 & 73,7 & & 52 & 29,7 & 123 & 70,3 & \\
\hline \multicolumn{11}{|l|}{ Procedência } \\
\hline Outros & 54 & 30,5 & 123 & 69,5 & 0,963 & 67 & 37,9 & 110 & 62,1 & 0,486 \\
\hline Salvador & 86 & 30,7 & 194 & 69,3 & & 97 & 34,6 & 183 & 65,4 & \\
\hline \multicolumn{11}{|l|}{ Com quem reside } \\
\hline Outros & 25 & 29,1 & 61 & 70,9 & 0,680 & 41 & 47,7 & 45 & 52,3 & $0,012^{*}$ \\
\hline Familiares & 116 & 31,4 & 254 & 68,6 & & 123 & 33,2 & 247 & 66,8 & \\
\hline
\end{tabular}

*Estatisticamente significante. 
república) e sozinho mostraram valor $p<0,005$. No modelo de Poisson robusto, a RP ajustada para sintomas de ansiedade manteve a associação estatisticamente significante para sexo (RP Aju = 1,31 IC95\% [1,17-1,47]), idade (RP Aju = 1,15 IC95\% [1,02-1,29]) e orientação sexual RP Aju = 1,90 IC95\% [1,26-2,86] (Tabela 2). A RP ajustada para sintomas de depressão manteve-se estatisticamente significante para sexo (RP Aju = 1,36 IC95\% [1,20-1,55]), raça/cor da pele (RP Aju = 1,96 IC95\% [1,29-3,04]) e orientação sexual (RP Aju = 1,19 IC95\% [1,03-1,36]) (Tabela 3). As prevalências de sintomas de ansiedade e depressão entre os ciclos básico, intermediário e internato não apresentaram diferença estatisticamente significante, respectivamente, $p=0,101$ e $p=0,601$.

Gráfico 1.Prevalência de sintomas de ansiedade e depressão em estudantes de Medicina - Salvador-BA, 2018

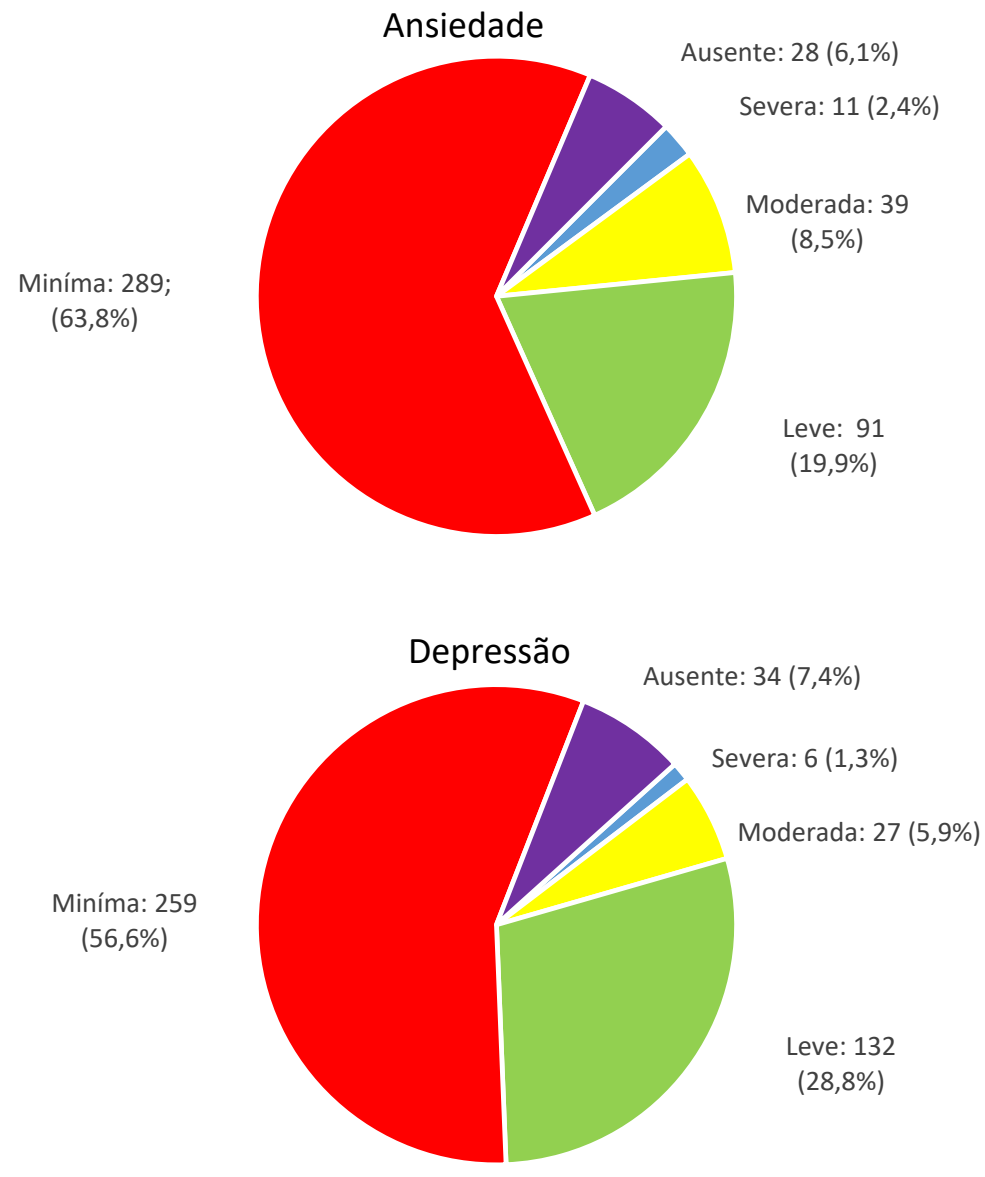

Tabela 2. Prevalência, razão de prevalência e intervalo de confiança cru e ajustado de sintomas de ansiedade em estudantes de Medicina segundo variáveis demográficas, sociais, econômica e comportamentais - Salvador-Bahia, 2018

\begin{tabular}{ccccc}
\hline Variável & Odds ratio cru & Intervalo de confiança & Odds ratio ajustado & Intervalo de confiança \\
\hline Sexo: Feminino & 2,39 & $1,53-3,74$ & 2,99 & $1,76-5,10$ \\
Idade: $<22$ anos & 1,72 & $1,13-2,62$ & 1,74 & $1,06-2,85$ \\
Orientação sexual: homo/bissexual & 3,85 & $1,86-7,99$ & 3,94 & $1,74-8,91$ \\
Situação afetiva & 0,76 & $0,51-1,14$ & - & - \\
Renda familiar & 1,45 & $0,92-2,29$ & - & - \\
\hline
\end{tabular}


Tabela 3. Prevalência, razão de prevalência e intervalo de confiança cru e ajustado de sintomas de depressão em estudantes de Medicina segundo variáveis demográficas, sociais, econômica e comportamentais - Salvador-Bahia, 2018

\begin{tabular}{ccccc}
\hline Variável & Odds ratio cru & Intervalo de confiança & Odds ratio ajustado & Intervalo de confiança \\
\hline Sexo: feminino & 2,30 & $1,51-3,50$ & 2,86 & $1,82-4,51$ \\
Orientação sexual: homo/bissexual & 3,40 & $1,63-7,11$ & 5,08 & $2,30-11,22$ \\
Raça/cor da pele: branca & 1,53 & $1,04-2,25$ & 1,58 & $1,05-2,37$ \\
Religião & 0,71 & $0,48-1,04$ & - & - \\
Renda familiar & 1,72 & $1,10-2,67$ & - & - \\
Com quem mora: familiares & 1,83 & $1,14-2,94$ & 1,94 & $1,17-3,21$ \\
\hline
\end{tabular}

\section{DISCUSSÃO}

A prevalência de sintomas de ansiedade entre estudantes de Medicina observada no presente estudo (30,8\%) foi inferior às encontradas por alguns autores - Ribeiro et al. ${ }^{27}$, Moutinho et al. ${ }^{28}$, Tabalipa et al. ${ }^{20}$, Ediz et al. ${ }^{19}$ e Costa et al. $^{23}$-, as quais variaram de $33,8 \%$ a $41,4 \%$. No entanto, esses mesmos autores apontaram para prevalências de sintomas de depressão entre $8,2 \%$ e 34,6\%, portanto inferiores à da presente investigação (36,0\%). Já se encontra estabelecido que sintomas de ansiedade e depressão estão presentes na vida cotidiana de milhões de pessoas no mundo, afetando tanto a saúde física como a mental, em especial estudantes universitários e aqueles da área da saúde.

As discordâncias nas prevalências encontradas na literatura podem ter ocorrido por conta das diferenças regionais e culturais da população de estudantes, das metodologias empregadas e dos tipos de questionário utilizados para obtenção dos dados. Entretanto, é consenso que o curso de Medicina é visto como um dos mais difíceis e que exige do aluno esforço concentrado na dedicação aos estudos e que há alta competitividade entre os estudantes ${ }^{29}$. A trajetória acadêmica do aluno de Medicina implica uma longa e cansativa jornada diária de atividades, o que envolve deslocamentos exaustivos que ocupam, inclusive, o seu tempo para atividades sociais e de lazer e até as horas de sono ${ }^{30,31}$. Outro fator extremamente importante é o contato com o sofrimento, a dor e até a morte do paciente, eventos que também causam tensão e estresse para os estudantes de Medicina. Toda essa situação de exaustão física e emocional dificulta o cuidado com a sua própria saúde, seja por falta de tempo ou por negligência do estudante e da faculdade, e torna elevado o risco de sintomas ansiosos/ depressivos e de desenvolvimento da síndrome de burnout ${ }^{32,33}$.

A maior prevalência de ansiedade e depressão no sexo feminino foi semelhante ao encontrado em outros estudos ${ }^{20,21,34}$ e consistente com o Mental Health Information, segundo o qual as desordens de ansiedade estiveram presentes no sexo feminino em $23,4 \%$ e $14,3 \%$ no masculino ${ }^{35}$, assim como com a declaração da OMS de que, mundialmente, a depressão é mais prevalente nas mulheres $(5,1 \%)$ do que nos homens $(3,6 \%)^{1}$. Tais diferenças, segundo a OMS, são consequência direta de violência de gênero, desvantagem socioeconômica, desigualdade de renda, status social baixo ou subordinado, cobranças sociais e responsabilidade pelo cuidado de outras pessoas, como a prole $^{36}$. Também outros fatores podem explicar, em parte, essa maior prevalência a exemplo de influências hormonais, já que a testosterona pode ter benefícios protetores contra a ansiedade e a depressão ${ }^{37}$.

A orientação sexual homo/bissexual apresentou maior prevalência de sintomas ansiosos e depressivos em relação à orientação heterossexual, que também está associada a esses desfechos. A angústia, negação, dúvida e até mesmo rejeição, por vezes da própria família, são problemas que esse grupo enfrenta desde a descoberta e aceitação da sua orientação sexual. Desse modo, há um aumento da insegurança e dos problemas psicossociais, e uma redução da autoestima, acarretando maior vulnerabilidade a problemas psicossociais, como uso de drogas, depressão e tentativas de suicídio ${ }^{38,39}$. Igualmente, maiores prevalências de sintomas depressivos ocorreram nos estudantes que se declararam da raça/cor da pele não branca (pardos e pretos), o que corrobora o resultado obtido por um estudo realizado com estudantes universitários da cidade do Rio de Janeiro ${ }^{40}$. Pesquisas apontam que fatores históricos como a falta de oportunidades, tanto educacionais como socioeconômicas, além dos estresses ligados aos papéis sociais e às experiências, como racismo e discriminação, são considerados importantes na maior prevalência entre pessoas negras do que entre as brancas ${ }^{41,42}$.

Os achados de Tabalipa et al. ${ }^{20}$, Baldassin et al..$^{43}$ e Bastos et al. $^{40}$, de frequência mais elevada de sintomas de ansiedade nos alunos do primeiro ao terceiro ano do curso de Medicina, justamente os mais jovens, foram similares aos de nosso estudo, o que é explicado pela melhor adaptação do aluno ao curso e pelo fortalecimento das relações interpessoais com colegas da turma, formando grupos que compartilham atividades afins, 
como estudo, lazer, viagens etc.

Apesar de não ter sido encontrada associação entre a sintomatologia de ansiedade e depressão e o fato de os discentes residirem com familiares, o apoio dos pais é uma fonte de força para os estudantes de Medicina enfrentarem dificuldades durante seus estudos e, ao mesmo tempo, também fortalece a autoconfiança deles ${ }^{17,33,44}$.

Consistentes com a literatura, sintomas ansiosos e depressivos foram mais frequentes nos alunos do terceiro, quinto e nono semestres do curso. Considerando os ciclos do curso, o básico apresentou prevalência maior do que os outros ciclos, dado semelhante ao encontrado por Costa et al. ${ }^{23}$. Ao ingressar na universidade, o aluno se depara com um ambiente completamente diferente do que vinha vivenciando nos anos anteriores de estudo ${ }^{45,46}$. Nos primeiros anos da faculdade, no período do curso básico, há a necessidade premente da integração com novos colegas e adaptação à nova metodologia de ensino, com excessiva quantidade de conteúdos teóricos, provas, seminários e outras demandas pedagógicas, que terminam estressando os alunos, resultando em exacerbação de sintomas ansiosos e depressivos ${ }^{44}$. Essa nova modalidade de ensino exige que o acadêmico desenvolva capacidades cognitivas e emocionais capazes de atender a essa nova demanda, o que repercute sobremaneira na qualidade de vida, sendo a escassez de tempo livre e o cansaço referidos pelos estudantes como os principais comprometedores da qualidade de vida ${ }^{47,48}$. Neste estudo, as prevalências, tanto de ansiedade como de depressão, apresentam também valores elevados no último ciclo, o internato. Acredita-se que o contato mais próximo com o paciente, muitas vezes incorporando as angústias, os medos, as ansiedades e a depressão dele, faz do próprio discente um prolongamento do paciente. Além disso, a expectativa pela finalização do curso, as provas de residência médica e a entrada no mercado de trabalho contribuem para o aumento dos sintomas de ansiedade e depressão ${ }^{48}$.

\section{CONCLUSÃO}

Vale referir que deve ser adotada alguma cautela na interpretação de alguns resultados deste estudo, pois algumas questões envolviam aspectos relacionados a "foro íntimo", como preferência sexual e renda familiar. Esses aspectos podem ter influenciado na veracidade das respostas dos estudantes. Por tratar-se de um estudo de prevalência, esta investigação não possibilita conclusões sobre causalidade, já que os resultados representam um panorama da magnitude dos sintomas de ansiedade e depressão e sua associação com fatores demográficos, sociais, econômicos e comportamentais. Estudos de acompanhamento adicionais são necessários para elucidar o curso da ansiedade e depressão nesse grupo populacional ao longo dos semestres letivos, visando apresentar uma melhor compreensão dos fatores que influenciam a saúde mental desses alunos e auxiliar no planejamento de intervenções para ajudá-los a lidar com os desafios enfrentados.

\section{CONTRIBUIÇÃO DOS AUTORES}

Bartira Oliveira Sacramento e Tassiana Lima dos Anjos participaram do planejamento do estudo, da coleta, análise e interpretação dos dados, e da redação e revisão final do artigo. Ana Gabriela Lopes Barbosa e Camila Fagundes Tavares participaram do planejamento do estudo e da coleta e análise dos dados. Juarez Pereira Dias participou do planejamento do estudo, da análise e interpretação dos dados, e da redação e revisão final do artigo.

\section{CONFLITO DE INTERESSES}

Os autores declaram não haver conflito de interesses neste estudo.

\section{FINANCIAMENTO}

Declaramos que não houve financiamento para a realização desta pesquisa.

\section{REFERÊNCIAS}

1. Global Health Estimates. Depression and other common mental disorders. Geneva: World Health Organization; 2017.

2. Nunes MA, Pinheiro AP, Bessel M, Brunoni AR, Kemp AH, Benseñor IM et al. Common mental disorders and sociodemographic characteristics: baseline findings of the Brazilian Longitudinal Study of Adult Health (ELSA-Brasil). Rev Bras Psiq. 2016;(38):91-97.

3. Risal A. Common mental disorders. Kathmandu Univ Med J. 2011;35(3):213-7.

4. Figueiredo MSL. Transtornos ansiosos e transtornos depressivos aspectos diagnósticos. Rev SPAGESP. 2000;1(1):89-97.

5. Associação Psiquiátrica Americana. Manual diagnóstico e estatístico de transtornos mentais. 5a ed. rev. (DSM-V-TR). Porto Alegre: Artmed; 2014.

6. Soares GB, Caponi S. Depression in focus: a study of the media discourse in the process of medicalization of life. Interface Comun Saúde Educ. 2011;15(37):437-46.

7. Organização Pan-Americana de Saúde. Folha informativa - Transtornos mentais. Washington, DC: Organização Pan-Americana de Saúde, Organização Mundial de Saúde; 2018.

8. Brasil. Diretrizes do NASF: Núcleo de Apoio à Saúde da Família. Brasília: Ministério da Saúde; 2010.

9. Patel V, Burns JK, Dhingra M, Tarver L, Kohrt BA, Lund C. Income inequality and depression: a systematic review and meta-analysis of the association and a scoping review of mechanisms. World Psychiatry. 2018;17:76-89.

10. Prince M, Patel V, Saxena S, Maj M, Maselko J, Phillips MR, et al. No health without mental health. Lancet. 2007;370:859-77.

11. Chisholm D, Sweeny K, Sheehan P, Rasmussen B, Smit F, Cuijpers P, et al. Scaling-up treatment of depression and anxiety: a global return on investment analysis. Lancet Psychiatry. 2016;3:415-24.

12. Santos GBV, Alves MCGP, Goldbaum M, Cesar CLG, Giovanini RJ. Prevalência de transtornos mentais comuns e fatores associados em moradores da área urbana de São Paulo, Brasil. Cad Saude Publica. 2019;35(11):e00236318. doi: 10.1590/0102-311X00236318. 
13. Moreira JKP, Bandeira M, Cardoso CS, Scalon JD. Prevalência de transtornos mentais comuns e fatores associados em uma população assistida por equipes do Programa Saúde da Família. J Bras Psiquiatr. 2011;60(3):221-6.

14. Harding TW, Arango MV, Baltazar J, Climent CE, Ibrahim HHA, LadridoIgnacio L, et al. Mental disorders in primary health care: a study of their frequency and diagnosis in four developing countries. Psychol Med. 1980;10:231-41.

15. Beck AT, Brown G, Epstein N, Steer RA. An inventory for measuring clinical anxiety: psychometric properties. J Consult Clin Psychol. 1988;56(6):893-7.

16. Puthran R, Zhang MWB, Tam WW, Ho RC. Prevalence of depression amongst medical students: a meta-analysis. Med Educ. 2016;50(4):456-68.

17. Pacheco JPG, Giacomin HT, Tam WW, Ribeiro TB, Arab C, Bezerra IM, et al. Mental health problems among medical students in Brazil: a systematic review and meta-analysis. Rev Bras Psiquiatr. 2017;39(4):369-78.

18. Fawzy M, Hamed SA. Prevalence of psychological stress, depression and anxiety among medical students in Egypt. Psychiatry Res. 2017;255:186-94.

19. Ediz B, Ozcakir A, Bilgel N. Depression and anxiety among medical students: examining scores of the Beck Depression and Anxiety Inventory and the depression anxiety and stress scale with student characteristics. Cogent Psychol. 2017;4(1):1-12.

20. Tabalipa FO, Souza MF, Pfützenreuter G, Lima VC, Traebert E, Traebert J. Prevalence of anxiety and depression among Medical students. Rev Bras Educ Med. 2015;39(3):388-94

21. Vasconcelos TC, Dias BRT, Andrade LR, Melo GF, Barbosa L, Souza E. Prevalência de sintomas de ansiedade e depressão em estudantes de Medicina. Rev Bras Educ Med. 2015;39(1):135-42.

22. Leão AM, Gomes IP, Ferreira MJM, Cavalcanti LPG. Prevalência e fatores associados à depressão e ansiedade entre estudantes universitários da área da saúde de um grande centro urbano do Nordeste do Brasil. Rev Bras Educ Med. 2018;42(4):55-65.

23. Costa DS, Medeiros NSB, Cordeiro RA, Frutuoso ES, Lopes JM, Moreira SNT. Sintomas de depressão, ansiedade e estresse em estudantes de Medicina e estratégias institucionais de enfrentamento. Rev Bras Educ Med. 2020;44(1):e040.

24. Quintão S, Delgado AR, Prieto G. Validity study of the Beck Anxiety Inventory (Portuguese version) by the Rasch Rating Scale Model. Psicol. Reflex Crit 2013;26(2):305-10.

25. Andrade I, Gorenstein C, Vieira Filho AH, Tung TC, Artes R. Psychometric properties of the Portuguese version of the State-Trait Anxiety Inventory applied to college students: factor analysis and relation to the Beck Depression Inventory. Braz J Med Biol Res. 2001;34(3):367-74.

26. Gomes-Oliveira MH, Gorenstein C, Lotufo Neto F, Andrade LH, Wang YP. Validation of the Brazilian Portuguese version of the Beck Depression Inventory-II in a community sample. Rev Bras Psiquiatr. 2012;34:389-94.

27. Ribeiro CF, Lemos CMC, Alt NN, Marins RLT, Corbiceiro WCH, Nascimento MI. Prevalence of and factors associated with depression and anxiety in Brazilian Medical students. Rev Bras Educ Med. 2020;44(1):e021.

28. Moutinho ILD, Maddalena NCP, Roland RK, Lucchetti ALG, Tibiriçá SHC, Ezequiel OS, et al. Depression, stress and anxiety in medical students: a cross-sectional comparison between students from different semesters. Rev Assoc Med Bras. 2017;63(1):21-8.

29. Arnold SS, Carvalho EA. Predomínio do estresse em acadêmicos de medicina. Rev Uningá Review. 2015;24(1):85-9.
30. Trindade LMDF, Vieira MJ. Curso de Medicina: motivações e expectativas de estudantes iniciantes. Rev Bras Educ Med. 2009;33(4):542-54.

31. Fiorotti KP, Rossoni RR, Miranda AE. Perfil do estudante de Medicina da Universidade Federal do Espírito Santo, 2007. Rev Bras Educ Med. 2010;34(3):355-62.

32. Benevides-Pereira AMT, Gonçalves MB. Transtornos emocionais e a formação em Medicina: um estudo longitudinal. Rev Bras Educ Med. 2009;33(1):10-23.

33. CMAJ. Medical schools addressing student anxiety, burnout and depression. CMAJ News. 2017;189:e1569-70

34. Sousa JM, Moreira CA, Telles-Correia D. Anxiety, depression and academic performance: a study amongst Portuguese Medical students versus nonMedical students. Acta Med Port. 2018;31(9):454-62.

35. National Institute of Mental Health. Mental Health Information [access in 20 apr 2020]. Available from: https://www.nimh.nih.gov/health/statistics/ index.shtml.

36. World Health Organization. Mental health [access in 20 apr 2020]. Available from: https://www.who.int/mental_health/prevention/genderwomen/en/.

37. McHenry J, Carrier N, Hull E, Kabbaj M. Sex differences in anxiety and depression: role of testosterone. Front Neuroendocrinol. 2014;35(1):42-57.

38. Everett BG. Optimal adolescent health to improve behavioral outcomes for LGBTQ youth. Salt Lake City: University of Utah; 2019.

39. Teixeira-Filho FS, Rondini CA. Ideações e tentativas de suicídio em adolescentes com práticas sexuais hetero e homoeróticas. Saúde Soc. 2012;21(3):651-67 [access in 20 apr 2020]. Available from: http://www. scielo.br/scielo.php?pid=S0104-12902012000300011\&script=sci_arttext.

40. Bastos JL, Barros AJD, Celeste RK, Paradies Y, Faerstein E. Age, class and race discrimination: their interactions and associations with mental health among Brazilian universitystudents. Cad Saude Publica.2014 Jan;30(1):17586 [access in 20 apr 2020]. Available from: http://www.scielo.br/scielo. php?script=sci_arttext\&pid=S0102-311X2014000100175\&lng=en.

41. Almeida-Filho N, Lessa I, Magalhães L, Araújo MJ, Aquino E, James SA, et al. Social inequality and depressive disorders in Bahia, Brazil: interactions of gender, ethnicity, and social class. Soc Sci Med. 2004;59(7):1339-53.

42. Smolen JR, Araújo EM. Raça/cor da pele e transtornos mentais no Brasil: uma revisão sistemática. Ciênc Saúde Colet. 2017;22(12):4021-30 [access in 20 apr 2020]. Available from: http://www.scielo.br/scielo.php?script=sci_ arttext\&pid=S1413-81232017021204021\&lng=pt.

43. Baldassin SP, Martins LC, Andrade AG. Traços de ansiedade entre estudantes de medicina. Arq Méd ABC. 2006;31(1):27-31.

44. Mustafa MB, Nasirb R, Yusooffb F. parental support, personality, selfefficacy and depression among medical students. Procedia Social and Behavioral Sciences. 2010;7(C):419-424.

45. Vallilo NG, Danzi Júnior R, Gobbo R, Novo NF, Hübner CK. Prevalência de sintomas depressivos em estudantes de Medicina. Rev Bras Clin Med. 2011;9(1):36-41.

46. Audy J. A inovação, o desenvolvimento e o papel da universidade. Estudos Avançados. 2017;31(90):75-87.

47. Paro CA, Bittencourt ZZLC. Qualidade de vida de graduandos da área da saúde. Rev Bras Educ Med. 2013;37(3):365-75.

48. Moran JM. Os novos espaços de atuação do professor com as tecnologias. Revista Diálogo Educacional. 2004;4(12):13-21. 